\title{
Acesso videolaparoscópico no tratamento cirúrgico do câncer colorretal: análise crítica
}

\author{
A. Habr-Gama, A.H. da Silva e Souza J R., S.E.A. Araujo \\ Disciplina de Coloproctologia, Departamento de Gastroenterologia da Faculdade de Medicina da Universidade de São Paulo, São Paulo, SP.
}

UNITERMOS: Colectomia. Câncer colorretal. Videolaparoscopia. Cólon. Reto.

KEY WORDS: Colorectal cancer. Video-laparoscopy. Laparoscopic colectomy.

\section{NTRODUÇÃO}

A perspectiva de oferecer aos doentes portadores de câncer do cólon e do reto tratamento cirúrgico menos invasivo, se comparado ao convencional - a saber, infligindo menor dor, menor permanência hospitalar e retorno mais precoce às atividades pessoais - , aliada ao rápido aperfei çoamento do instrumental laparoscópico, levou diversos autores a provar a exeqüibilidade da cirurgia colorretal por acesso videolaparoscópico em experiências iniciais.

A crescente demanda, por parte dos doentes, por procedimentos realizados às custas de menor trauma responde, atualmente, pela força motriz dos esfor ços nesta área. As experiências iniciais publicadas na literatura, no que concerne ao tratamento curativo do câncer de cól on e reto, produziram, até o momento, dados cientificamente questionáveis. Em oposição ao que acontece para a colecistectomia, existem, até o momento, poucas evidências demonstrando a superioridade da ressecção do câncer colorretal com finalidade curativa por laparoscopia, quando comparada à realizada por via convencional. Para os doentes candidatos às operações de caráter paliativo, as vantagens estão bem estabelecidas, à semelhança do que ocorre para as lesões benignas colorretais e para a realização de procedimentos, tais como estomias, reconstituição do trânsito ou retopexias. Para as operações com objetivo de cura, as prerrogativas essenciais em oncologia, como a ligadura na origem dos vasos mesentéricos inferiores, a ressecção em monobloco, asséptica e com margem de segurança, e a linfadenectomia das estações de drenagem com os propósitos de estadiamento e prognóstico, devem ser rigorosamente respeitadas, uma vez que estão integradas aos procedimentos realizados pela via convencional. Não obstante, é ine- gável que a maioria dos doentes que foram operados por acesso videolaparoscópico relatados na literatura se beneficiou do tratamento oferecido.

Constitui objetivo do presente trabal ho expor as evidências obtidas até o momento, favoráveis e contrárias ao tratamento radical do câncer colorretal por acesso videolaparoscópico. Desta forma, os autores acreditam contribuir para a racionalização dos esforços em pesquisas nesta área.

\section{ESTADIAME NTO INTRA-OPERATÓRIO}

Analogamente ao fato de não ser possível a realização de operação do tipo no touch por acesso vi deolaparoscópico, a pal pação intra-operatória do parênquima hepático encontra-se inviabilizada, comprometendo a compl eta avaliação do fígado e de outras vísceras sólidas. Em contrapartida, a inspeção hepática das faces diafragmática e visceral por acesso videolaparoscópi co encontra-se preservada para a mai oria dos casos quando é possível valer-se de pinças auxiliares para a exposição das superfícies hepáticas e lise de bridas - nos casos de cirurgias prévias em andar superior do abdome.

Sabe-se que a ultra-sonografia intra-operatória tem-se destacado como método efi caz de detecção de metástases hepáticas, podendo identificar lesões de até $5 \mathrm{~mm}$ de diâmetro que são, freqüentemente, imperceptíveis à ultra-sonografia transabdominal, tomografia computadorizada e, mesmo, à pal pação intra-operatória ${ }^{1}$. O emprego da ultra-sonografia intra-operatória pode resultar em incremento diagnóstico de lesões secundárias hepáticas da ordem de $10 \%{ }^{2}$.

Diante dessas evidências, iniciam-se experiências individuais de diversos autores no sentido de determinar o potencial diagnóstico da ultra-sonografia laparoscópica. Além de seu emprego na abordagem pré-operatória do carcinoma hepatocelular em cirróticos, vem sendo utilizada no estadiamento intra-operatório de pacientes submetidos a colectomia Iaparoscópica por câncer. Resultados iniciais advindos do emprego dessa técnica são animadores ${ }^{3}$. 


\section{EXTENSÃO DA RESSE CÇÃO INTESTINAL}

No que se refere ao tratamento radical da doença maligna colorretal, a ressecção de segmento de cólon por acesso videolaparoscópico suficientemente longo às necessidades oncológicas mostrouse tecnicamente exeqüível em diversas séries publicadas na literatura ${ }^{4-9}$, com excelente campo operatório e facilidade de mobilização do cól on por via laparoscópi ca. E stá demonstrada a obtenção de margens de ressecção idênticas em colectomias por câncer realizadas por acesso convencional e videol aparoscópi $\mathrm{co}^{10}$.

O exame endoscópico do cólon reveste-se de mai or importância diante dos procedimentos realizados por acesso videolaparoscópico. A realização de colonoscopia pré ou peri-operatória pode localizar a lesão e proceder à sua impregnação por substâncias como a tinta da china, o azul de metileno e o carvão ativado ${ }^{11}$, permitindo rápido e acurado método de identificação da lesão sob vi são laparoscópica, eliminando a possibilidade de ressecção equivocada de segmento de cólon livre de doença.

\section{LIGADURA DA ARTÉ RIA MESE NTÉ RICA INFERIOR}

A nal ogamente ao que acontece quando da avaliação da extensão da ressecção col orretal para doença maligna, observa-se que a ligadura da artéria mesentérica inferior junto à sua origem na aorta vem sendo realizada de forma similar à realizada por via convencional nas ressecções por acesso videolaparoscópico, no tratamento radical da doença maligna do intestino grosso $0^{6,10,12-14}$.

De forma elegante e inédita, Decanini et al. ${ }^{15}$ obti veram comprimento médio de cinco milímetros para o coto remanescente da artéria mesentérica inferior, em operações de amputação do reto por acesso videolaparoscópico realizadas em cadáveres frescos, subseqüentemente necropsiados com vistas a verificar se foram obedecidos os critérios de radicalidade oncológi ca estabel ecidos para essa operação.

\section{EXTENSÃO DA DISSE CÇÃO LINFONODAL}

A exérese cirúrgica das estações de drenagem linfática do segmento colorretal acometido por câncer constitui, hoje, princípio básico do tratamento cirúrgico das doenças malignas.

Antes de qualquer análise dos relatos a respeito da extensão da ressecção linfonodal realizada por acesso videolaparoscópico, cabem as considerações:
- o número de linfonodos encontrados em um espécime cirúrgico depende da perseveração do patologista na dissecção e identificação deles;

- existe enorme variabilidade no número de linfonodos que acompanham as peças cirúrgicas, mesmo quando da análise das operações convencionais $s^{16}$;

- o relato puro e simples do número absoluto de linfonodos extraídos pode não ser útil à avaliação da vali dade da operação, uma vez que o significado prognóstico de linfonodo pericól i co acometi do difere daquele associado a achado de linfonodo apical (junto à ligadura vascular) acometido;

- o número de linfonodos presentes no espécime cirúrgico parece não afetar o prognóstico, segundo alguns autores ${ }^{17}$, pois, na verdade, o número de linfonodos que efetivamente são prognósticos é aquele que permaneceu no doente operado.

Desta forma, ainda que esse número seja de discutível valor no estabelecimento do prognóstico, séries na literatura demonstram que o número de linfonodos obtidos nas ressecções por acesso videolaparoscópico corresponde ao obtido por ressecções convencionais ${ }^{6,10,18}$.

\section{RECIDIVAS TUMORAIS PRECOCES}

Existem, até o momento, relatos da ocorrência de recidivas tumorais em sítios de punção de trocartes após operações curativas para câncer colorretal e realizadas por acesso videolaparoscópico, totalizando pelo menos 30 casos.

Algumas considerações devem ser realizadas a respeito desse preocupante fenômeno:

- nem todas as recidivas tumorais verificaramse em incisão por onde foi retirada a peça;

- as recidivas aconteceram mesmo nos casos em que a peça foi retirada envolta em saco plástico protetor;

- ocorreram recidivas tumorais mesmo nos casos cujo tumor era restrito à parede do cólon (Dukes A);

- a recidiva neoplásica em sítios de punção de trocartes tem sido verificada não somente para os casos de neoplasia colorretal.

A ocorrência de recidiva tumoral em incisão cirúrgica, em operações realizadas por acesso convencional para o tratamento do câncer colorretal, conforme rel ato disponível na literatura, é de $1 \%{ }^{19}$. Tal freqüência pode estar subestimada, considerando-se a probabilidade de ocorrência de subregistro desses casos quando considerados como decorrência da evolução natural do câncer de intestino grosso. Há, portanto, que se estabel ecer, de forma prospectiva e diante das recidivas ocorridas 
em doentes operados por laparoscopia, um registro para a devida quantificação desses casos.

Conforme estimativa realizada por Wexner ${ }^{20}$, acredita-se que essa inci dência para a intervenção realizada por acesso videolaparoscópico gire em torno de $4 \%$ e que, também, há indícios de que esteja subestimada.

A interferência do gás carbônico, tanto na disseminação de cél ulas tumorais na circulação como no seu crescimento, tem sido considerada como possível explicação para o aumento das recorrências. Conforme demonstrado por J ones et al. em estudo experimental ${ }^{21}$, o estabel ecimento de pneumoperitôni o com gás carbônico aumenta a ocorrência de implantes peritoniais e em sítios de trocartes após a infusão peritonial de células de câncer de cólon humano em hamsters. Em contrapartida, a ocorrência de implantes neoplásicos em sítos de punção de trocartes após operações de pleuroscopia por malignidade torácica restringe o alcance de eventuais conclusões advindas do estudo conduzido por J ones et al.

Ainda, foi observado por Harmon ${ }^{22}$ et al. que, na colectomia laparoscópica, os níveis medidos de interleucina- 6 foram mais baixos do que os verificados na colectomia convencional, resposta esta independente do tempo operatório e da perda sanguínea. Portanto, na via laparoscópica, a resposta ao trauma cirúrgico é menor, com redução dos efeitos imunossupressores, o que representa benefício em relação às complicações infecciosas, e, talvez, aumentando a sobrevida após a ressecção do intestino grosso por câncer.

\section{DURAÇÃO DA OPERAÇÃO}

Diversas séries na literatura demonstram que os procedimentos realizados por acesso videol aparoscópi co têm duração superi or aos realizados por acesso convencional 7,14,23,24. A permanência do doente por intervalo de tempo prol ongado na posição de Trendelenburg necessária à dissecção colorretal e com abdome insuflado por pneumoperitônio pode levar a um aumento na incidência de fenômenos tromboembólicos, ainda que não demonstrada na literatura. Além disto, a significante absorção de gás carbônico que ocorre nestes procedimentos prolongados pode levar a hipercapnia de difícil manejo anestésico, ocasionando, por vezes, a conversão $o^{13}$ do procedimento e subseqüente aumento do tempo operatório. A hipercapnia, nessas ocasiões, pode ser extremamente prejudicial a doentes com função pulmonar comprometida ${ }^{25}$. O treinamento do cirurgião na nova metodologia, seguramente, contribui para o encurtamento do tempo operatório; entretanto, isto não é conseguido sem muito esforço e perseverança. O julgamento do momento oportuno para a conversão ao método aberto é importante, a conversão devendo ser realizada sempre que houver dificuldades em prosseguir na dissecção alargada.

\section{DURAÇÃO DO ÍLEO E INTENSIDADE DA DOR NO PÓS-OPERATÓRIO}

As impressões clínicas iniciais s, $^{6,26-28}$ advindas de estudos retrospectivos utilizando grupos-controle históricos de pacientes operados pelo acesso convencional de que doentes submetidos a operações colorretais realizadas por acesso videolaparoscópi co apresentariam período de conval escença mais curto, associado a menor dor e menor tempo de íleo, quando comparados a doentes operados por via convencional, foram confirmadas por pelo menos dois estudos prospectivos ${ }^{10,23}$.

Tais estudos demonstram menor intensidade da dor em doentes operados por acesso videolaparoscópico, traduzida pelo menor consumo de medicação analgésica opiácea ou não no período pósoperatório nesses doentes, quando comparados aos operados por acesso convencional.

Esses achados corroboram as opiniões iniciais de que tais doentes, dado que sentem menor dor em função da menor ou ausente incisão abdominal, e da não-utilização de afastadores, deambulam mais e consomem menos medicação opiácea e, portanto, recuperam-se mais rapidamente, apresentando retorno da função intestinal mais precocemente.

\section{MORBIDADE E MORTALIDADE}

A morbidade associada a procedimentos realizados por acesso videolaparoscópico (a análise em separado dos procedimentos realizados exclusivamente para o tratamento do câncer col orretal ainda não se encontra disponível na literatura), conforme revisão recente ${ }^{29}$ envolvendo 460 casos, é de 16,9\%. A morbidade associada a procedimentos realizados por acesso convencional, e disponível na literatura, é de $37,2 \%{ }^{30}$. Menor morbidade associada a procedimentos realizados por acesso videolaparoscópico também foi comprovada por estudos prospecti$\operatorname{vos}^{31,32}$. Dado interessante advindo de estudo prospectivo revela que a perda sanguínea associada aos procedimentos laparoscópicos é menor do que a associada aos procedimentos realizados por via convencional ${ }^{23}$. Tal fato se revela de maior importância quando estão estudadas evidências de pior prognóstico associado a hemotransfusões durante as operações curativas para câncer colorretal ${ }^{33}$. 
No que se refere à morbi dade intrínseca associada aos procedimentos realizados por acesso videolaparoscópico, uma série de complicações até o momento incomuns foram observadas. A corrida desenfreada - levada a cabo por cirurgiões por vezes não afeitos à dissecção colorretal convencional - no sentido de obter o pi oneirismo na realização desta ou daquel a operação consignou morbidade injustificável pela aclamada curva de aprendizado, a saber: enterotomias inadvertidas ${ }^{8,14}$; lesão vascular intra-abdominal ${ }^{8}$; lesão ureteral ${ }^{5}$; herniação por sítio de punção de trocarte ${ }^{18}$; fístulas de anastomose ${ }^{8}$; ressecção equivocada de segmento de cól on livre de doença ${ }^{5,6}$.

E $m$ recente questionário enviado a membros da Sociedade Americana de Cirurgiões de Cólon e Reto ${ }^{34}$, foram relatados 12 casos de ressecção de segmento de cól on são, isto é, livre de doença; e essas intervenções, portanto, demandaram procedimento cirúrgico complementar destinado a ressecar o segmento doente e objeto da intervenção inicial.

Ainda que a ressecção equi vocada possa ser evitada por exame endoscópico pré ou perioperatório, a necessidade compulsória deste para a real ização de uma operação segura é declarada desvantagem do método laparoscópico.

Fato interessante neste mesmo inquérito advém da observação de que, apesar de $71 \%$ dos cirurgiões entrevistados indicarem operação por acesso videolaparoscópico para um caso de câncer de cólon ou reto, apenas $15 \%$ destes se submeteriam a operação laparoscópica, caso portassem câncer de ceco, e somente $6 \%$ deles seriam operados por laparoscopia, caso se apresentassem com câncer do reto.

A mortalidade advinda da análise dos mesmos dados relativos a operações por laparoscopia foi de $1 \%$ e comparável à descrita para os procedimentos realizados por via convencional ${ }^{30,35}-2-5 \%$.

\section{DURAÇÃO DA PERMANÊ NCIA HOSPITALAR}

Diversos relatos, resultados de observações retrospectivas, bem como prospectivas, na literatura obtiveram tempo de internação hospitalar menor associado aos procedimentos realizados por acesso videolaparoscópico, quando comparados aos procedimentos realizados por via convencional 7,10,18,23,29,32. O menor tempo de internação hospitalar parece ser decorrente de menor tempo de íleo pós-operatório, menor dor e realimentação precoce; entretanto, esses achados não foram verificados uniformemente entre os relatos. Com exceção de apenas uma sér $\mathrm{e}^{36}$, todos os estudos na literatura evidenciam tempo de internação reduzido.

A perspectiva de oferecer aos pacientes tempo de internação abreviado constitui alternativa desejável e atraente ao cirurgião e ao próprio paciente, pois reduz o impacto social e o custo do tratamento cirúrgico. Entretanto, a necessidade de vigilância pós-operatória objetivando diagnóstico precoce das complicações relacionadas às anastomoses colorretais - fístulas e deiscência - parece ser responsável pela pequena diferença no que se refere à duração da internação hospitalar para pacientes operados pelo acesso convencional e laparoscópico. Permanece em aberto a discussão sobre este aspecto, uma vez que a opção de observação domi ciliar do paciente operado por acesso videolaparoscópico e portador de anastomose col or retal parece não gozar da simpatia da maioria dos cirugiões de cól on e reto.

\section{CUSTOS}

A colectomia laparoscópica tem custos hospitalares el evados, se comparada à realizada por via convencional. Tal fato é atribuível, analogamente, ao que ocorrre para a herniorrafia inguinal, ao tempo operatório ainda al ongado dessas intervenções e ao alto custo do material empregado. Ainda que tais números não estejam disponíveis para a realidade nacional, esses achados constituem, per se, empeciIho à aplicação do acesso videol aparoscópico no tratamento das afecções colorretais benignas, opção atraente e que vem ganhando, paulatinamente, a preferência dos pacientes e dos coloproctologistas.

Confirmando as impressões acima, Hoffman et al. ${ }^{14}$, em experiência inicial composta por 80 pacientes submetidos a colectomia realizada por acesso videolaparoscópico e comparado-os com 53 pacientes submetidos a colectomia convencional, obtiveram tempo operatório maior e maior custo associado aos procedimentos realizados por Iaparoscopia.

\section{RESULTADO ESTÉTICO}

Em que pese não ser objetivo primordial do tratamento radical do câncer do intestino grosso, a cirurgia colorretal laparoscópica apresenta excelente efeito estético decorrente tanto da menor incisão realizada, quando dos procedimentos ditos "assistidos", como da ausência desta nos procedimentos verdadeiramente laparoscópicos, a exemplo da amputação do reto. A observação de alguns autores de que a menor taxa de morbidade associada a tais procedimentos pode advir da menor incisão, entretanto, demanda, ainda, estudos idealizados com a finalidade de medir qual o percentual da menor morbidade observada que pode ser atribuível somente à menor incisão ou acesso cirúrgico. 


\section{CONCLUSÕES}

Diante das evidências apresentadas, os autores concluem que há necessidade de estudos prospectivos randomizados comparando o acesso videolaparoscópico ao convencional, no tratamento radical do câncer de cól on e reto. Os estudos disponíveis são, em sua maioria, retrospectivos e, certamente, não contrapõem grupos comparáveis, podendo, desta forma, apenas sugerir conclusões e, timidamente, servir de suporte a condutas clínicas.

Enquanto a cirurgia colorretal videolaparoscópica continuar a ser empregada como tratamento radical do câncer em casuísticas isol adas e realizadas fora dos centros de referência e, portanto, desvinculados de protocolos estabelecidos, não se vislumbrarão condições éticas mínimas para a realização de estudos prospectivos e randomizados, conduzidos por grupos experientes - que não contenham exclusões -, imprescindíveis para a elucidação dos aspectos controvertidos desta nova via de acesso.

\section{REFERÊNCIAS BIBLIOGRÁFICAS}

1. Charnley RM, Morris DL, Dennison AR et al. Detection of colorectal liver metastases using intraoperative ultrassonography. Br J Surg 1991; 78: 45-8.

2. Parker GA, Lawrence J r W, Horsley J S III et al. Intraoperative ultrassound of the liver affects operative decision making. Ann Surg 1989; 209: 569-77.

3. Bezzi M, Merlino R, Orsi $F$ et al. Laparoscopic sonography during abdominal laparoscopic surgery: technique and imaging findings. Am J Radiol 1995; 165: 1.193-8.

4. Habr-Gama A, Sousa J r AHS, Araujo SA. Análise crítica das intervenções laparoscópicas sobre o cólon. In: CBC Vídeocirurgia, São Paulo, Roca, 1993; 377-89.

5. Larach SW, Salomon MC, Williamson PR et al. Laparoscopic assisted colectomy: experience during the learning curve. Coloproctol ogy 1993; 1: 38-41.

6. Monson J R, Darzi A, Declan Carey P, Guillou PJ . Prospective evaluation of laparoscopic assisted colectomy in an unselected group of patients. Lancet 1992; 340: 831-2.

7. Musser DJ , Boorse RC, Madera F, Reed III J F. Laparoscopic colectomy: at what cost ? Surg Laparosc E ndosc 1994; 4(1): 1-5.

8. Wexner SD, Cohen SM, J ohansen OB et al. Laparoscopic colorectal surgery: a prospective assessment and current perspective. Br J Surg 1993; 80: 1.602-5.

9. Zucker KA, Pitcher DE, Martin DT, Ford RS. Laparoscopic assisted colon resection. Surg Endosc 1994; 8: 12-8.

10. Van YeTM, Cattey RP, Henry LG. Laparoscopically assisted col on resections compare favorably with open technique. Surg Laparosc Endosc 1994; 4(1): 25-31.

11. Kitamura K, Yamane T, Oyama T et al. Rapid and accurate method for delineating cancer lesions in laparoscopic colectomy using activated carbon injection. J Surg Oncol 1995; 58: 31-4.

12. Corbitt J D. Preliminary experience with Iaparoscopic-guided colectomy. Surg Laparosc Endosc 1992; 2(1): 79-81.

13. Franklin ME, Rosenthal D, Norem RF. Prospective evalua- tion of laparoscopic col on resection versus open colon resection for adenocarcinoma. Surg Endosc 1995; 9: 811-6.

14. Hoffman J C, Baker J W, Fitchett CW, Vansant J H. Laparoscopic-assisted colectomy: initial experience. Ann Surg 1994; 219(6): 732-43.

15. Decanini C, Milson J W, Böhm B, Fazio VW. Laparoscopic oncologic abdominoperineal resection. Dis Colon Rectum 1994; 37: 552-8.

16. Cohen SM, Wexner SD, Schmitt SL et al. Does xylene mesenteric fat clearance improve lymph node harvest after colon resection? Eur J Surg 1994; 160(12): 693-7.

17. Shida J , Ban K, Matsumoto M et al. Prognostic significance of location of lymph nodal metastases in colorectal cancer. Dis Col on Rectum 1992; 35: 1.046-50.

18. Falk PM, Beart RW, Wexner SD et al. Laparoscopic colectomy: a critical appraisal. Dis Col on Rectum 1993; 36: 28-34.

19. Hughes ES, M cDermott FT, Poliglase AI, J ohnson WR. Tumor recurrence in the abdominal wall scar after large bowel cancer surgery. Dis Col on Rectum 1983; 26: 571-2.

20. Wexner SD, Cohen SM. Port site metastases after laparoscopic col orectal surgery for cure of malignancy. Br J Surg 1995; 82: 295-8.

21. J ones DB, Guo LW, Reinhard MK et al. I mpact of pneumoperitoneum on trocar site implantation of colon cancer in hamster model. Dis Col on Rectum 1995; 38: 1.182-8.

22. Harmon G, Senagore AJ, Kilbride MJ, Warzynski MJ . Interleukin- 6 response $x$ laparoscopic and open colectomy. Dis Col on Rectum 1994; 37: 754-9.

23. RamosJ M, Beart RW, Goes R, Ortega AE, Schlinkert RT. Role of laparoscopy in col orectal surgery: a prospective evaluation of 200 cases. Dis Col on Rectum 1995; 38: 494-501.

24. Wexner SD, J ohansen OB, Nogueras J J , J agelman DG. Laparoscopic total abdominal colectomy. A prospective trial. Dis Colon Rectum 1992; 35: 651-5.

25. McMahon AJ , Baxter J N, O'Dwyer PJ . Preventing complications of laparoscopy. Br J Surg 1993; 80: 1.593-4.

26. Phillips EH, Franklin M, Carrol BJ et al. Laparoscopic colectomy. Ann Surg 1992; 216: 703-7.

27. Puente I, Sosa J L, Sleeman D et al. Laparoscopic assisted colorectal surgery. J Laparoendosc Surg 1994; 4: 1-7.

28. Scoggin SD, Frazee RC, Snyder SK et al. Laparoscopic assisted bowel surgery. Dis Col on Rectum 1993; 36: 747-50.

29. Mathis CR, Mac F ayden J r BV. Laparoscopic col orectal resection: a review of the current experience. Int Surg 1994; 79: 221-2.

30. Bokey EL, Chapuis PH, F ung C et al. Postoperative morbidity and mortality following resection of the colon and the rectum for cancer. Dis Col on Rectum 1995; 38: 480-7.

31. Reiver $\mathrm{D}, \mathrm{K}$ mi ot WA, Cohen SM et al. A prospective comparison of laparoscopic and open procedures in colorectal surgery. Dis Colon Rectum 1994; 37 (4): 22 [Resumo].

32. Ortega A, Beart RW, Anthone G, Schlinkert R. Laparoscopic bowel resection: a consecutiveseries. Dis Col on Rectum 1994; 37(4): 22 [Resumo].

33. Sugarbaker PH, Corlew S. Influence of surgical techniques on survival in patients with colorectal cancer. Dis Colon Rectum 1982; 25: 545-57.

34. Cohen SM, Wexner SD. Laparoscopic colorectal surgery: are we being honest with our patients? Dis Colon Rectum 1995; 38: 723-7.

35. Goligher J . Results of operations for large bowel cancer. In: Decosse JJ (ed). New York, Churchill-Livingstone, 1981; 154-65.

36. Wexner SD, J ohansen OB. Laparoscopic bowel resection: advantages and limitations. Ann Med 1992; 24: 105-10. 